\title{
Chapter 10 \\ Voronoi Tessellations and Their Application to Climate and Global Modeling
}

Lili Ju, Todd Ringler, Max Gunzburger

\begin{abstract}
We review the use of Voronoi tessellations for grid generation, especially on the whole sphere or in regions on the sphere. Voronoi tessellations and the corresponding Delaunay tessellations in regions and surfaces on Euclidean space are defined and properties they possess that make them well-suited for grid generation purposes are discussed, as are algorithms for their construction. This is followed by a more detailed look at one very special type of Voronoi tessellation, the centroidal Voronoi tessellation (CVT). After defining them, discussing some of their properties, and presenting algorithms for their construction, we illustrate the use of CVTs for producing both quasi-uniform and variable resolution meshes in the plane and on the sphere. Finally, we briefly discuss the computational solution of model equations based on CVTs on the sphere.
\end{abstract}

\section{Introduction}

Given two sets $A$ and $B$ and a distance metric $d(a, b)$ defined for $a \in A$ and $b \in B$, a Voronoi diagram or tessellation is a subdivision of $A$ into subsets, each of which contains the objects in $A$ that are closer, with respect to the distance metric, to one object in $B$ than to any other object in $B$. Although Voronoi tessellations can be defined for a wide variety of sets and metrics, of interest here is the situation for

Lili Ju

Department of Mathematics, University of South Carolina, Columbia, SC 29208, USA, e-mail: ju@math.sc.edu

Todd Ringler

T-3 Fluid Dynamics Group, Theoretical Division, Los Alamos National Laboratory, Los Alamos, NM 87545, USA, e-mail: ringler@lanl.gov

Max Gunzburger

Department of Scientific Computing, Florida State University, Tallahassee, FL 32306-4120, USA, e-mail: gunzburg@fsu.edu 
which the set $A$ is a region or surface in Euclidean space, $B$ is a finite set of points also in Euclidean space, and the metric is the Euclidean distance.

Voronoi tessellation have a long history, probably because Voronoi-like arrangements often appear in nature. Voronoi-like tessellations appeared in 1644 in the work of Decartes on the distribution of matter in the cosmic region near our sun. The first systematic treatment of what we now call Voronoi tessellations was given by Dirichlet (Dirichlet, 1850) in his study of two- and three-dimensional quadratic forms, i.e., homogeneous, multivariate polynomials of degree two; hence, Voronoi regions are often referred to as Dirichlet cells. Voronoi (Voronoi, 1907) generalized the work of Dirichlet to arbitrary dimensions, again using what are now referred to as Voronoi tessellations or diagrams.

The first documented application of Voronoi tessellations appeared in the classic treatise of Snow (Snow, 1855) on the 1854 cholera epidemic in London in which he demonstrated that proximity to a particular well was strongly correlated to deaths due to the disease. Voronoi tessellations have continued to be very useful in the social sciences, e.g., in the study of dialect variations, demographics, territorial systems, economics, and markets. Starting in the late 19th century and continuing to this day, Voronoi tessellations have also been used in crystallography, especially in the study of space-filling polyhedra, although, in this setting, various other names have been used to denote Voronoi regions, e.g., stereohedra, fundamental area, sphere of influence, domain of action, and plesiohedra.

It is not surprising, due to their ubiquity and usefulness, that throughout the 20th century, Voronoi tessellations were rediscovered many times. As a result, Voronoi regions have been called by many different names. Thiessen polygons refer to the work of Theissen on developing more accurate estimates for the average rainfall in a region. Area of influence polygons was a term coined in connection with the processing of data about ore distributions obtained from boreholes. Wigner-Seitz regions, domain of an atom, and Meijering cells were terms that arose from work on crystal lattices and the Voronoi cell of the reciprocal crystal lattice is referred to as the Brillouin zone (Kittel, 2004; Ziman, 1979). In the study of codes by, e.g., Shannon, Voronoi cells are called maximum likelihood regions (Weaver and Shannon, 1963). The field of ecology gave rise to two more alternate labels: area potentially available and plant polygons for a Voronoi region associated with a particular tree or plant. Capillary domains refers to Voronoi regions in a tissue based on the centers of capillaries.

For a long time, the routine use of Voronoi tessellations in applications was hindered by the lack of efficient means for their construction. This situation has now been remedied, at least in two and three dimensions. Voronoi tessellations also became closely intertwined with computational geometry. For example, Shamos and Hoey (Shamos and Hoey, 1975) not only provided an algorithm for constructing Voronoi tessellations, but also showed how they could be used to answer several fundamental questions in computational geometry. 
Delaunay tessellations, ${ }^{1}$ the dual concept to Voronoi tessellations, also have a long history and have been called by other names. They originated with Voronoi (Voronoi, 1908) who called them the ensemble (L) of simplices. Delone (Delaunay, $1928,1934)$ was the first to define the tessellations bearing his name ${ }^{2}$ in terms of empty spheres; he referred to them in terminology similar to that of Voronoi and, even today, some refer to Delaunay tessellations as L-partitions. The name Delaunay was first associated with Delaunay triangulations by Rogers (Rogers, 1964). Delaunay tessellations have also proven to be very useful, especially for grid generation.

The first applications of Voronoi tessellations to global atmospheric modeling were made by Williamson (Williamson, 1968) and Sadourny et al. (Sadourny et al, 1968) wherein the barotropic vorticity equation was integrated forward in time. Neither Williamson nor Sadourny referred to their meshes as Voronoi tessellations; Williamson referred to the underlying tessellation as a "geodesic grid," a colloquialism that is used in much of the literature discussing the use of Voronoi tessellations in global climate modeling. ${ }^{3}$ Both of these efforts produced promising results as compared to other models available at the time. The reason for their success was really due to not having a longitudinal polar filter which distorted the earlier solutions on latitude-longitude grids, or to not using a reduced grid which also distorted the solutions. In addition, it helped as well that the discrete formation of the Jacobian put forth by Arakawa (Arakawa, 1966) could be readily translated to their respective "geodesic grids." Williamson (Williamson, 1970) continued this line of research with the integration of the shallow-water system in primitive variable form. While Williamson's tessellation was extremely uniform, in a global sense, as compared to the latitude-longitude meshes being using in other model development efforts (Kasahara and Washington, 1967), the truncation error analysis by Williamson clearly re-

\footnotetext{
${ }^{1}$ Delaunay tessellations are often referred to as Delaunay triangulations because, in two dimensions, they consist of a triangulation of the points that generate the Voronoi tessellation. We choose to refer to them as Delaunay tessellations to emphasize the fact that the concept of a dual to Voronoi tessellations is quite general and not limited to two dimensions. When dealing with twodimensional settings, we will however, call them Delaunay triangulations.

2 Delone was a Russian number theorist who used the spelling Delaunay when writing papers in French or German. He was also the first to coin both the descriptors "Dirichlet domains" and "Voronoi regions."

3 Adjectives such as "geodesic," "bisection," and "icosahedral" are often used to describe grids on the sphere. However, there seems to be a lack of consistency about what these qualifiers mean. In this paper, we use the following terminology.

Geodesic grids refer to any grid on the sphere such that the edges of the grid cells are geodesic arcs, i.e., arcs of great circles. According to this definition, all Voronoi grids on the sphere are, by construction, geodesic grids.

Bisection grids refer to any grid constructed through repeated bisection of a platonic solid having vertices on the sphere and edges projected onto the sphere. Bisection grids are by construction geodesic grids. One may also define a bisection grid by repeated bisection of the Delaunay triangulation corresponding to the platonic solid.

Octahedral-bisection grids refer to bisection grids that are based on the platonic octahedra having 12 pentagonal faces. Note that this grid is often referred to as a "geodesic grid" or a "bisection grid" but here we make a finder distinction between these terminologies.
} 
flected the fact that the Voronoi tessellation was less uniform in a local sense; the discrete operators used in (Williamson, 1970) resulted in first-order truncation errors that could quickly corrupt the solution. Williamson's barotropic primitive equation model was discretized using a collocated grid where thickness and velocity reside at the same location. ${ }^{4}$ Since a collocated grid makes little (if any) use of the dual mesh, it is not clear if this geodesic grid was or was not a Voronoi tessellation.

Following Williamson (Williamson, 1970), the idea of solving the barotropic primitive equations based on a Voronoi tessellation was essentially abandoned for fifteen years. It appears that this idea did not gain traction for two reasons. First, global spectral models emerged as a superior choice to their finite-volume or finitedifference counterparts because they are based on the natural polar filter so to speak and have no pole problem. Their spectral accuracy and the reintroduction of the fast Fourier transform (Cooley and Tukey, 1965) also contributed significantly. Second, while numerical schemes situated on latitude-longitude meshes were burdened with truncation errors comparable to those found by Williamson (Williamson, 1970), progress toward methods to mitigate the impact of these errors on the longterm stability of simulations was much more rapid for quadrilateral meshes; see, e.g., (Arakawa and Lamb, 1977). Unfortunately, the numerical methods developed for the solution of the barotropic primitive equations on quadrilateral meshes did not readily translate to Voronoi tessellations. For example, while C-grid staggered quadrilateral meshes were essentially operational by the mid 1970s, a comparable C-grid scheme for general Voronoi tessellations was not derived until Thuburn et. al. (Thuburn et al, 2009) in 2009.

Because it appeared, at least at the time, that Voronoi tessellations were not well suited for the integration of the primitive equations, when this idea was revisited by Masuda and Ohnishi (Masuda and Ohnishi, 1986) they chose a different system of equations to discretize. Masuda and Ohnishi formulated the shallow-water system in vorticity and divergence variables, instead of primitive variables. In this approach, the thickness, vorticity, and divergence are collocated at the center of each Voronoi cell. Other similar work on solving shallow water equations based on Voronoi mesh was done by Augenbaum, et. al. (Augenbaum, 1984; Augenbaum et al, 1985). Randall (Randall, 1994) would later show that the collocation of variables in the vorticity-divergence system, termed the Z-grid, leads to a simulation of geostrophic adjustment that is better than any of the other staggerings based on primitive variables. The superior simulation of geostropic adjustment along with the direct control over the evolution of vorticity led to robust simulations of the shallowwater system. Heikes and Randall (Heikes and Randall, 1995a,b) continued this line of research with the implementation of a geometric multigrid solver to mitigate the cost associated with solving the vorticity-divergence system. In turn, this work led to the creation, by Ringler et. al. (Ringler et al, 2000) in 2000, of the first global atmosphere dynamical core situated on a Voronoi tessellation.

The demonstration that Voronoi tessellations could be used to successfully model global atmosphere dynamics created considerable interest. By and large, all global

4 The collocated grid was later named the "A-Grid" in (Arakawa and Lamb, 1977). 
atmosphere models using finite-volume methods were based on latitude-longitude grids. With no satisfactory solutions to overcome the grid singularities present at the poles of latitude-longitude grids, the quasi-uniform grid offered by Voronoi tessellations was a compelling alternative. This stimulated research toward finding numerical schemes based on primitive variables that would essentially translate the A-, Band C-grid staggerings from quadrilateral meshes to Voronoi tessellations. The collocated, A-grid staggering, first proposed by Williamson (Williamson, 1970), was successfully implemented by Tomita et. al. (Tomita et al, 2001). That effort resulted in the first ever global cloud resolving simulation by Tomita et al. (Tomita et al, 2005). It is important to note that (Tomita et al, 2001) and (Tomita et al, 2005) do not employ a Voronoi tessellation since the location of the cell vertices are placed at the barycenter ${ }^{5}$ of the Delaunay triangulation, instead of the circumcenter of the Delaunay triangulation. As a result, the powerful results that follows from a Voronoi tessellation are not immediately applicable to their mesh. The B-grid staggering was successfully developed for Voronoi tessellations by Ringler and Randall (Ringler and Randall, 2002). It is only at this point, fully two decades after the energy and potential enstrophy conserving schemes for quadrilateral grids were derived (Arakawa and Lamb, 1981), that the numerical methods on Voronoi tessellations are comparable to their quadrilateral counterparts.

With the successful implementation of both the discrete vorticity-divergence system and various discrete forms of the primitive equation system on quasi-uniform Voronoi tessellations, attention is now turning toward the use of variable resolution Voronoi tessellations. During this process we are essentially revisiting the truncation error problems that Williamson (Williamson, 1970) identified four decades ago when using quasi-uniform Voronoi tessellations. When pairing low-order, finitevolume methods with variable resolution Voronoi tessellations, truncation error will be increased, at least locally, in the regions of mesh transition. To overcome the challenge presented by this truncation error behavior, we see three routes forward. First, increase the accuracy of the underlying finite-volume method to reduce truncation error to acceptable levels; this approach was successfully employed in (Du et al, 2003b; Du and Ju, 2005; Weller, 2009; Weller and Weller, 2008). Second, develop numerical schemes that respect both geostrophic adjustment and the need for nonlinear stability, even when the mesh is highly distorted; this approach has been developed by Thuburn et. al. (Thuburn et al, 2009) and Ringler et. al. (Ringler et al, 2010). And finally, we can attempt to optimize the quality of the variable resolution meshes in order to limit the extent of the problem. In the end, some combination of these three approaches will likely lead to the creation of a variable-resolution global climate system model.

This chapter is focused on the mesh generation aspect of Voronoi tessellations and, more importantly, the inherent properties that these meshes are guaranteed to possess. We first provide a mathematical description of Voronoi tessellations and their Delaunay triangulation counterparts. This is followed by a detailed analysis of

\footnotetext{
5 The barycenter is the center of mass; thus, for a triangle, the barycenter is at the intersection of the three lines joining the vertices and the centers of the opposite sides whereas the circumcenter is at the intersection of the perpendicular bisectors of the three sides.
} 
one very special type of Voronoi tessellation, the centroidal Voronoi tessellation. We then explore the properties of centroidal Voronoi tessellations when producing both quasi-uniform and variable resolution meshes. Finally, we briefly discuss the numerical implementation of models using centroidal Voronoi tessellations. We defer until Section 3 a discussion about why centroidal Voronoi tessellations are especially well suited as a basis for grid generation.

\section{Voronoi and Delaunay tessellations}

\subsection{Definitions and properties}

We are given an open bounded domain $\Omega \in \mathbb{R}^{d}$ and a set of distinct points $\left\{\mathbf{x}_{i}\right\}_{i=1}^{n} \subset$ $\Omega$. For each point $\mathbf{x}_{i}, i=1, \ldots, n$, the corresponding Voronoi region $V_{i}, i=1, \ldots, n$, is defined by

$$
V_{i}=\left\{\mathbf{x} \in \Omega \mid\left\|\mathbf{x}-\mathbf{x}_{i}\right\|<\left\|\mathbf{x}-\mathbf{x}_{j}\right\| \quad \text { for } j=1, \cdots, n \text { and } j \neq i\right\}
$$

where $\|\cdot\|$ denotes the Euclidean distance (the $L^{2}$ metric) in $\mathbb{R}^{d}$. Clearly $V_{i} \cap V_{j}=\emptyset$ for $i \neq j$, and $\cup_{i=1}^{n} \bar{V}_{i}=\bar{\Omega}$ so that $\left\{V_{i}\right\}_{i=1}^{n}$ is a tessellation of $\Omega$. We refer to $\left\{V_{i}\right\}_{i=1}^{n}$ as the Voronoi tessellation or Voronoi diagram of $\Omega$ (Okabe et al, 2000) associated with the point set $\left\{\mathbf{x}_{i}\right\}_{i=1}^{n}$. A point $\mathbf{x}_{i}$ is called a generator; a subdomain $V_{i} \subset \Omega$ is referred to as the Voronoi region or Voronoi cell corresponding to the generator $\mathbf{x}_{i}$.

It is clear that, except for "sides" that are part of the boundary of $\Omega$, Voronoi regions $\left\{V_{i}\right\}_{i=1}^{n}$ are polygons in two dimensions and polyhedra in three dimensions. Figure 1 (upper left) presents a Voronoi tessellation of the unit square in two dimensions corresponding to ten randomly selected generators. It is guaranteed that the line segment connecting two neighbor generators is orthogonal to the shared edge/face and is bisected by that edge/face.

The dual of a Voronoi tessellation in the graph-theoretical sense (i.e., by connecting all pair of neighbor generators) is called a Delaunay tessellation or, in two dimensions, a Delaunay triangulation (Okabe et al, 2000) associated with the point set $\left\{\mathbf{x}_{i}\right\}_{i=1}^{n}$. Elements of a Delaunay tessellation consist of triangles in two dimensions and tetrahedra in three dimensions. The Delaunay triangulation corresponding to the above ten generators is shown in Figure 1 (top right). Note that each triangle of the Delaunay triangulation is associated with a single vertex of its dual Voronoi tessellation. That Voronoi vertex is located at the center of the circumscribed circle of the triangle; see an illustration in Figure 1 (bottom). Each cell edge of the Voronoi tessellations is uniquely associated with one cell edge of the dual Delaunay triangulation; each pair of edges are orthogonal, but do not necessarily intersect. If the pair of edges do intersect (or if the lines segments are extended to a point where

\footnotetext{
${ }^{6}$ For the open region $\Omega, \bar{\Omega}$ denotes its closure, i.e., $\Omega$ together with its boundary points.
} 


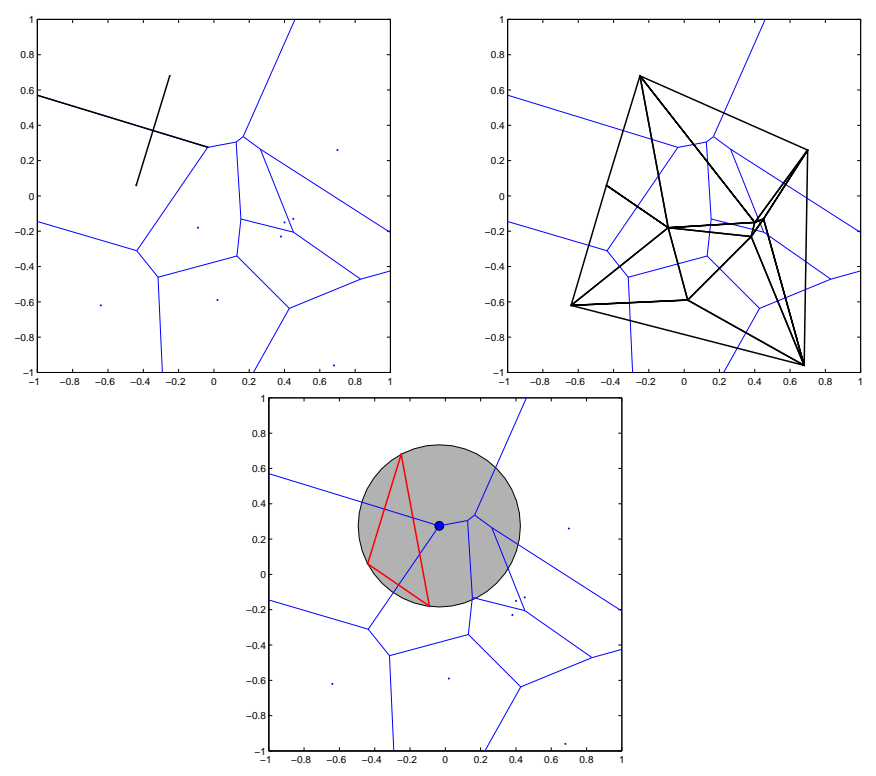

Fig. 1 The Voronoi tessellation of the unit square corresponding to 10 randomly selected generators. Top-left: the bisection property; top-right: the corresponding Delaunay triangulation; bottom: the circumcicle property.

they intersect), then the intersection point will bisect the line segment connecting generators.

In two dimensions, the Delaunay triangulation maximizes the minimum angle, i.e., compared to any other triangulation of the points, the smallest angle in the Delaunay triangulation is at least as large as the smallest angle in any other. This property does not hold in higher dimensions. Note also that for a given set of generators, the Voronoi tessellation is always unique; however, the Delaunay tessellation may not be unique in certain special situations, e.g., when four generators in two dimensions form a rectangle that does not contain any other generator.

Voronoi and Delaunay tessellations of a general surface or manifold also have been widely studied in the field of computer graphics; see, e.g., (Boissonnat and Oudot, 2005). In particular, spherical Voronoi tessellation and Delaunay triangulation and related algorithms are developed in (Renka, 1997).

\subsection{Construction algorithms}

For a given set of distinct points $\left\{\mathbf{x}_{i}\right\}_{i=1}^{n} \subset \Omega$, the construction of the corresponding Voronoi tessellation and Delaunay triangulation in Euclidean space has been well studied in past decades; see (Okabe et al, 2000). Note that some algorithms directly compute the Delaunay tessellation whereas others compute the Voronoi tessellation. 
As illustrated in Figure 1, a property of the Delaunay triangulation is that the circle circumscribing any Delaunay triangle does not contain any other generators in its interior. This is an important property because it allows the use of a flipping technique. If a triangle is non-Delaunay, we can flip one of its edges; see Figure 2 for an illustration. This leads to the simple flip algorithm: construct any triangulation of the points, and then flip edges until no triangle is non-Delaunay. Unfortunately, this can take $O\left(n^{2}\right)$ edge flips. It is worth noting that this edge-flipping technique does not directly extend to three or higher dimensions; on the other hand, the circumcircle property itself does generalize, e.g., to circumspheres of the Delaunay tetrahedra in three dimensions, and some topological operations analogous to flipping have been proposed and discussed in three dimensions (Freitag and Ollivier-Gooch, 1997; Du and Wang, 2003; Alliez et al, 2005).
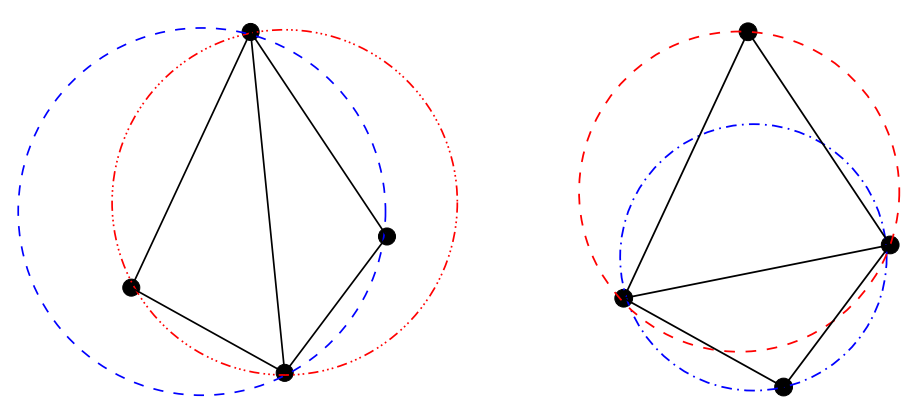

Fig. 2 The flipping operation. Left: the triangulation does not meet the Delaunay condition, i.e., the circumcircles contain more than three points; right: flipping the common edge produces a Delaunay triangulation for the four points.

A usually more efficient way to construct the Delaunay triangulation is to repeatedly add one vertex at a time and then re-triangulate the affected parts of the graph. When a point $\mathbf{x}_{i}$ is added, the triangle containing $\mathbf{x}_{i}$ is split into three triangles and then the flip algorithm is applied. This procedure is called the incremental algorithm. It takes $O(n)$ time to search through all the triangles to find the one that contains $\mathbf{x}_{i}$, after which we potentially flip in every triangle. The overall runtime is theoretically $O\left(n^{2}\right)$ (Guiba et al, 1992), but often in practice this algorithm has better than expected performance (Bentley et al, 1980). While the technique extends to higher dimension, the complexity could grow exponentially in the dimension, even if the final Delaunay triangulation is small (Edelsbrunner and Shah, 1996).

An efficient divide and conquer algorithm (Lee and Schachter, 1980; Guibas and Stolfi, 1985) for constructing a Voronoi tessellation of a given set of generators in the plane is defined as follows. One recursively draws a line to split the generators into two sets having roughly the same number of points. Voronoi tessellations of the two subsets are separately constructed. Then, a piecewise linear dividing line between the two subsets is determined. Each segment of this line is itself a segment of the perpendicular bisector corresponding to two generators belonging to different subsets. Then, all edges or part of edges from the Voronoi tessellations of each sub- 
set that lie on the opposite side of the dividing line are deleted. Finally, the Voronoi tessellation of the original set of generators is given by the union of the remaining edge segments of the Voronoi tessellations of the two subsets and the piecewise linear dividing line. See Figure 3 for illustrative sketches of the divide and conquer algorithm. Carefully implemented, this divide and conquer method for constructing a Voronoi tessellation of a given set of generators has complexity $O(n \log n)$. A divide and conquer paradigm for constructing a triangulation in $d$-dimensions was developed in (Cignoni et al, 1998).
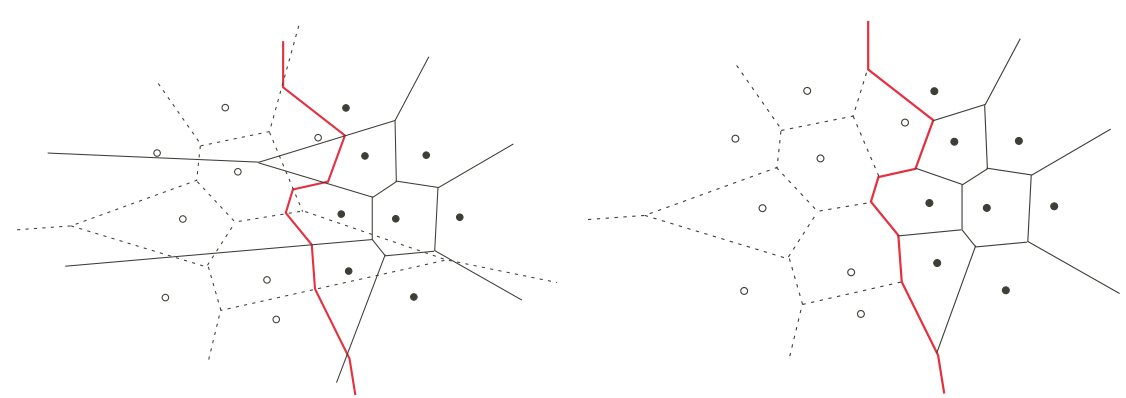

Fig. 3 Left: the divide-and-conquer algorithm after the given generators are divided into two subsets (the open and filled circles), the two Voronoi tessellations of the subsets have been constructed (the dashed lines and thin solid lines), and the piecewise linear dividing line has been determined (the thick, red lines). Right: the Voronoi tessellation of all the generators found by deleting appropriate portions of the Voronoi tessellations of the two subsets.

Another efficient algorithm, Fortune's sweep line algorithm (Fortune, 1986), is based on the sweep line technique (Sedgewick, 1983) and involves not only a sweep line, but also a beach line that actually consists of parabolic arcs. Without loss of generality, one can assume that the sweep line is vertical and that it moves from left to right. Generators to the right of the sweep line have yet to be considered. The beach line is to the left of the sweep line. It is defined as follows: first, for each generator to the left of the sweep line whose Voronoi region has yet to be completely determined, one defines the parabola that separates the points that are closer to the sweep line from those that are closer the generator; then, the beach line is determined as the right-most points in the union of the parabolas. Clearly, a vertex of the beach line is equidistant from the two generators corresponding to the parabolas meeting at that vertex. Thus, as the sweep line moves from left to right, the vertices of the beach line move along the edges of the Voronoi tessellation. A parabolic arc is added to the beach line whenever the sweep line passes a new generator; an arc is removed from the beach line whenever the Voronoi cell for the corresponding generator has been completely determined. The latter situation occurs whenever the sweep line is tangent to a circle passing through three generators whose parabolas form consecutive arcs of the beach line. See Figure 4 for an illustrative sketch for Fortune's algorithm. Carefully implemented, Fortune's algorithm for constructing a Voronoi tessellation of a given set of generators has complexity $O(n \log n)$. 


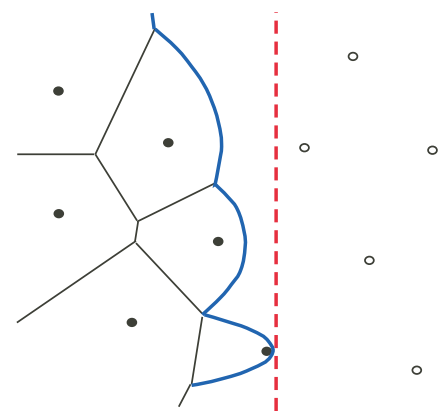

Fig. 4 Set-up in Fortune's algorithm. The red, dashed straight line is the sweep line that is moving from left to right; the blue, piecewise parabolic curve is the beach line. The filled circles are the generators already visited by the sweep line whereas the open circles are generators yet to be visited. The thin black lines are edges or edge segments of Voronoi regions already constructed.

Finally, we mention "convex hull" algorithms (Chynoweth and Sewell, 1990) for, e.g., Delaunay tessellation construction in Euclidean regions. For example, in the two-dimensional case, one can vertically project the generators from their plane onto a paraboloidal surface whose axis is perpendicular to that plane. The lower boundary of the convex hull of the points on the paraboloid is generally a triangulated shell whose vertical projection back onto the original plane gives the Delaunay triangulation. This geometrical characterization also explains the circumcircle property mentioned above. The plane of any triangular facet of the assumed convex shell intersects the paraboloid on a closed curve whose projection is that projected triangle's circumcircle. Thus, other generators lying strictly inside that circle would have to correspond to points of the paraboloid that necessarily lie outside the putative convex hull, in violation of the original assumption that a convex hull was constructed. See (Chynoweth and Sewell, 1990; Sewell, 2002) for detailed discussions on this characterization.

\section{Centroidal Voronoi tessellations}

Centroidal Voronoi tessellations (CVTs) are special Voronoi tessellations having the property that the generators of the Voronoi tessellation are also the centers of mass (or centroids or barycenters), with respect to a given density function, of the corresponding Voronoi regions. CVT methodologies produce high-quality point distributions in regions and surfaces in $\mathbb{R}^{d}$ or within sets of discrete data. In the latter context and in its simplest form, CVT reduces to the well-known $k$-means clustering algorithm (Gersho and Gray, 1992; Hartigan, 1975; Kanungo et al, 2002). The dual tessellation corresponding to a centroidal Voronoi tessellation is referred to as a centroidal Voronoi Delaunay tessellation (CVDT). 
CVTs and CVDTs possess certain properties, which we discuss below, that make them very well suited for grid generation which is a focus of this paper. In addition, in (Nguyen et al, 2009), several quality measures were used to effect a quantitative comparison of uniform triangular mesh generators in convex and non-convex planar regions; it was found that CVDTs result in higher quality meshes compared to those constructed using most other algorithms, with only the method given in (Persson and Strang, 2004) that uses spring dynamics being somewhat competitive.

Although uniform CVT-based grids have been shown to be competitive with (or even better than) other uniform mesh generators in planar, three-dimensional, and spherical regions, perhaps they have even greater utility for the construction of nonuniform meshes. For one thing, through a point density function, CVT grid generation methodologies allow for a simple means of controlling the local grid size; moreover, the density function can easily be connected to error estimators, resulting in effective adaptive refinement strategies (Ju et al, 2002b). For another thing, CVTbased grids feature smooth transitions from coarse to fine grids; see Section 4.1.2 for an illustration. Smooth grid transitions can greatly reduce deleterious effects, e.g., non-physical wave reflections, that can occur if grid sizes change abruptly.

\subsection{Definitions and properties}

Given a density function $\rho(\mathbf{x}) \geq 0$ defined on $\Omega$, for any region $V \subset \Omega$, the standard mass center (or centroid) $\mathbf{x}^{*}$ of $V$ is given by

$$
\mathbf{x}^{*}=\frac{\int_{V} \mathbf{x} \rho(\mathbf{x}) \mathrm{d} \mathbf{x}}{\int_{V} \rho(\mathbf{x}) \mathrm{d} \mathbf{x}} .
$$

Note that it is often required that $\rho$ is integrable with respect to $\Omega$ and the volume of the set $\{\mathbf{x} \mid \rho(\mathbf{x})=0\}$ is zero in order to make sure (2) is well defined in practice. A special family of Voronoi tessellations are defined as follows.

Definition 1 (Du et al, 1999) Given a density function $\rho(\mathbf{x})$ defined on $\Omega$, we refer to a Voronoi tessellation $\left\{\left(\mathbf{x}_{i}, V_{i}\right)\right\}_{i=1}^{n}$ of $\Omega$ as a centroidal Voronoi tessellation (CVT) if and only if the points $\left\{\mathbf{x}_{i}\right\}_{i=1}^{n}$ which serve as the generators of the associated Voronoi regions $\left\{V_{i}\right\}_{i=1}^{n}$ are also the centroids, with respect to $\rho(\mathbf{x})$, of those regions, i.e., if and only if we have that $\mathbf{x}_{i}=\mathbf{x}_{i}^{*}$ for $i=1, \ldots, n$. The corresponding dual triangulation is called a centroidal Voronoi Delaunay tessellation (CVDT).

A generic Voronoi tessellation does not in general satisfy the CVT property; see Figure 5 for an illustration as well as for an illustration of CVT. On the other hand, given a density function $\rho$ and the number $n$ of generators, the CVT of a domain always exists, although it may not be unique.

CVTs possess an optimization property that can be used as a basis for various extensions. Given any set of points $\widetilde{\mathbf{X}}=\left\{\widetilde{\mathbf{x}}_{i}\right\}_{i=1}^{n}$ in $\Omega$ and any tessellation $\widetilde{\mathbf{V}}=$ 

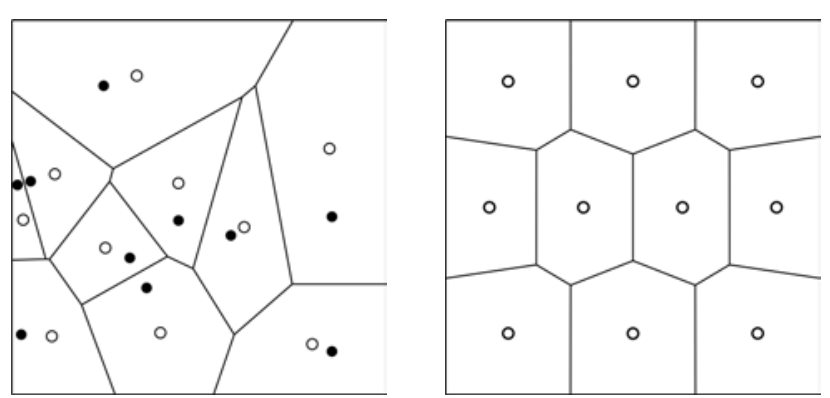

Fig. 5 (Du et al, 1999) Left: a Voronoi tessellation of the unit square with 10 randomly selected generators (the filled circles); the open circles denote the centroids of the Voronoi polygons with respect to a uniform density; the centroids do not coincide with the generators. Right: a 10-generator centroidal Voronoi tessellation of the square for a uniform density; the generators and centroids coincide.

$\left\{\widetilde{V}_{i}\right\}_{i=1}^{n}$ of $\Omega$, define a clustering energy by ${ }^{7}$

$$
\mathscr{K}(\widetilde{\mathbf{X}}, \widetilde{\mathbf{V}})=\sum_{i=1}^{n} \int_{\widetilde{V}_{i}} \rho(\mathbf{x})\left\|\mathbf{x}-\widetilde{\mathbf{x}}_{i}\right\|^{2} \mathrm{~d} \mathbf{x} .
$$

Then, it can be shown that $\mathscr{K}$ is minimized only if $\left\{\left(\widetilde{\mathbf{x}}_{i}, \widetilde{V}_{i}\right)\right\}_{i=1}^{n}$ forms a CVT ${ }^{8}$ of $\Omega$. Note that if $\left\{\left(\mathbf{x}_{i}, V_{i}\right)\right\}_{i=1}^{n}$ forms a CVT, it does not necessarily minimize $\mathscr{K}$, e.g., it may define a saddle point (Du et al, 1999) of (3). In many applications, the clustering energy functional $\mathscr{K}$ is often naturally associated with quantities such as quantization error, variance, and cost.

Asymptotically, as the number of generators becomes larger and larger, Gersho's conjecture (Gersho, 1979) states that, locally, the optimal CVT (in the sense of minimizing the clustering energy) under the Euclidean metric forms a regular tessellation consisting of the replication of a single polytope whose shape depends only on the spatial dimension. ${ }^{9}$ The regular hexagon provides a confirmation of the conjecture in two dimensions for the constant density case (Newman, 1982). For the threedimensional case and a constant density function, it has been proved (Barnes and Sloane, 1983; Du and Wang, 2005) that among all lattice-based CVTs,${ }^{10}$ the CVT corresponding to the body-centered cubic lattice for which the Voronoi regions are the space-filling truncated octahedra is the optimal one. For more general, nonlattice cases and for non-constant densities, the question remains open, although

\footnotetext{
${ }^{7}$ Note that, a priori, $\mathbf{V}$ need not be a Voronoi tessellation and $\mathbf{x}_{i}$ need not be in $V_{i}$.

${ }^{8}$ In fact, this can be used as an analytical definition of CVTs alternate to the geometric definition given in Definition 1.

${ }^{9}$ In other words, Gersho's conjecture states that, at least for smooth density functions, if the number of generators $n$ is large enough and one focuses on a small enough region, then a CVT appears to be a uniform tessellation involving congruent polytopes.

${ }^{10}$ A lattice-based CVT is one whose generators are located on a lattice so that the Voronoi regions form congruent polytopes.
} 
extensive numerical simulations given in (Du and Wang, 2005) demonstrated that the truncated octahedra remains the likely candidate. It is interesting to note that, in two dimensions, Gersho's conjecture implies that the dual Delaunay triangulation asymptotically consists of a replications of a single polygon, namely congruent equilateral triangles. In three dimensions, the dual Delaunay tessellation cannot consist of congruent equilateral tetrahedra because the latter cannot cover three space.

\subsubsection{Centroidal Voronoi tessellations of surfaces}

Extensions of the VT and CVT concept to surfaces (or manifolds) are possible; for example, tessellations of surfaces under the Euclidean metric are considered in (Du et al, 2003a). Suppose that $\Omega$ is a compact and continuous hypersurface in $\mathbb{R}^{d+1}$. Then, for any subregion $V \subset \Omega$, we call $\mathbf{x}^{c}$ a constrained mass center of $V$ if it is a solution of the problem:

$$
\text { find } \mathbf{x}^{c} \text { such that } \int_{V} \rho(\mathbf{y})\|\mathbf{y}-\mathbf{x}\|^{2} \mathrm{~d} \mathbf{y} \quad \text { is minimized over } \mathbf{x} \in V \text {. }
$$

Existence of minimizers of the problem (4) can be easily demonstrated using the continuity and compactness of the objective function; however, solutions may not be unique. It is worth noting that if $\Omega$ is a flat surface, then $\mathbf{x}^{c}$ coincides with $\mathbf{x}^{*}$, the standard center of mass center of $V$. If we replace $\mathbf{x}_{i}^{*}$ in Definition 1 by $\mathbf{x}_{i}^{c}$, then the resulting Voronoi tessellation $\left\{\left(\mathbf{x}_{i}, V_{i}\right)\right\}_{i=1}^{n}$ of the surface $\Omega$ is called a constrained centroidal Voronoi tessellation (CCVT) (Du et al, 2003a), and its dual tessellation is called a constrained centroidal Voronoi Delaunay triangulation (CCVDT). In particular, when $\Omega$ is the surface of a sphere, we call $\left\{\left(\mathbf{x}_{i}, V_{i}\right)\right\}_{i=1}^{n}$ a spherical centroidal Voronoi tessellation (SCVT). Figure 6 presents an illustration of non-centroidal and centroidal Voronoi tessellations of the sphere.
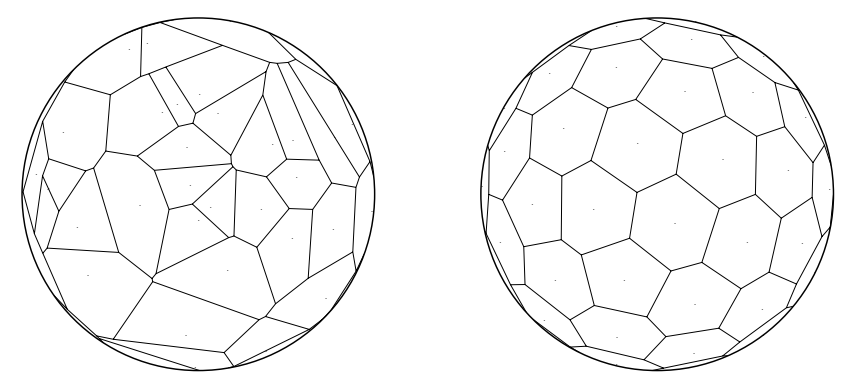

Fig. 6 (Du et al, 2003a) Left: A spherical Voronoi tessellation with 64 randomly selected generators. Right: a 64-generator spherical centroidal Voronoi tessellation for the uniform density.

The calculation of the constrained centroid $\mathbf{x}^{c}$ for any given subregion $V$ of a smooth surface $\Omega$ can be effected using Newton's method or a damped Newton's 
method (Ju, 2007). However, a more direct and less costly approach may be used instead. One can first compute the standard centroid $\mathbf{x}^{*}$ of the subregion $V$ as defined in (2). Note that, in general, the standard centroid $\mathbf{x}^{*}$ of $V$ does not lie on the surface $\Omega$; for example, for a region on the sphere, $\mathbf{x}^{*}$ is inside the sphere. Then, as is shown in (Du et al, 2003a), the constrained centroid $\mathbf{x}^{c}$ of $V \in \Omega$ can be found by projecting $\mathbf{x}^{*}$ onto $\Omega$ along the normal direction at $\mathbf{x}^{c}$. In particular, if $V$ is a subset of the surface of a sphere of radius $r$, we have that its constrained center of mass is given by $\mathbf{x}^{c}=r \mathbf{x}^{*} /\left\|\mathbf{x}^{*}\right\|$.

\subsection{Algorithms for constructing CVTs}

CVTs can be constructed either using probabilistic methods typified by MacQueen's random algorithm (MacQueen, 1967) (which simply alternates between sampling and averaging points) or deterministic methods typified by Lloyd's method (Lloyd, 1982) (which simply alternates between constructing Voronoi tessellations and mass centroids). Due to its effectiveness and simplicity, much attention has been focused on Lloyd's method.

Algorithm 1 (Lloyd's Method) Given a domain $\Omega$, a density function $\rho$ defined on $\Omega$, and a positive integer $n$ (the number of generators).

1. Select an initial set of $n$ points $\left\{\mathbf{x}_{i}\right\}_{i=1}^{n}$ on $\Omega$.

2. Construct the Voronoi regions $\left\{V_{i}\right\}_{i=1}^{n}$ of $\Omega$ associated with $\left\{\mathbf{x}_{i}\right\}_{i=1}^{n}$.

3. Determine the centroids (or constrained centroids), with respect to the given density function, of the Voronoi regions $\left\{V_{i}\right\}_{i=1}^{n}$; these centroids form the new set of points $\left\{\mathbf{x}_{i}\right\}_{i=1}^{n}$; if $\Omega$ is a hypersurface, then $\mathbf{x}_{i}$ must be projected onto $\Omega$.

4. If the new points meet some convergence criterion, return $\left\{\left(\mathbf{x}_{i}, V_{i}\right)\right\}_{i=1}^{n}$ and terminate; otherwise, go to Step 2.

It has been shown (Du et al, 1999) that the energy $\mathscr{K}$ associated with the Voronoi tessellation decreases monotonically during the Lloyd iteration until a CVT is reached. Some convergence analyses of the Lloyd's method are given in (Du et al, 2006; Emelianenko et al, 2008).

In Step 1 of Algorithm 1, the initial set of points can be selected at random. However, because Lloyd's method only finds local minima of the clustering energy $\mathscr{K}$, the generator positions of the final CVT produced is affected by the initial distribution of generators. ${ }^{11}$ Therefore, in some situations, one may want to use less noisy starting conditions; an example is given in Section 4.1.1.

For the second step, the methods described in Section 2.2 can be applied. There also exist software packages that may be used for Voronoi tessellation construction. For example, on the sphere, there is the STRIPACK package (Renka, 1997).

\footnotetext{
${ }^{11}$ This is true for other CVT construction methods because, invariably, they only find local minima of the clustering energy.
} 
The computation of centroids of the Voronoi regions in the third step of Algorithm 1 can be effected by first decomposing each Voronoi region into a set of triangles/tetrahedra and then using a high-order quadrature rule for triangles/tetrahedra to approximate integrals appearing in (2). Note that if the region of interest is a surface, e.g., part of the sphere or the whole sphere, then this step also includes the projection of the Euclidean centroid onto the surface; see Section 3.1.1.

For the fourth step, an example of a stopping criterion is if some measure, e.g., the root mean square, of the movement of the generators from one iteration to the next is smaller than a prescribed tolerance; alternately, one can stop if the change in the (computable) clustering energy is smaller than a prescribed tolerance.

A probabilistic version of a generalized Lloyd's method was proposed in (Ju et al, 2002a) together with its parallel implementation. ${ }^{12}$

Algorithm 2 (Probabilistic Generalized Lloyd's Method) Given a domain $\Omega, a$ density function $\rho$ defined on $\Omega$, and a positive integer $n$.

1. Choose a positive integer $q$ (the number of sampling points per iteration) and constants $\left\{\alpha_{i}, \beta_{i}\right\}_{i=1}^{2}$ such that $\alpha_{2}>0, \beta_{2}>0, \alpha_{1}+\alpha_{2}=1$, and $\beta_{1}+\beta_{2}=1$; choose an initial set of $n$ points $\left\{\mathbf{x}_{i}\right\}_{i=1}^{n}$; set $j_{i}=1$ for $i=1, \ldots, n$.

2. Choose $q$ sample points $\left\{\mathbf{y}_{r}\right\}_{r=1}^{q}$ in $\Omega$ at random, e.g., by a Monte Carlo method, with the density function $\rho(\mathbf{x})$ acting as the probability density function. 3. For $i=1, \ldots, n$, gather together in the set $W_{i}$ all sampled points $\mathbf{y}_{r}$ closest to $\mathbf{x}_{i}$ among $\left\{\mathbf{x}_{i}\right\}_{i=1}^{n}$, i.e., all sampled points in the Voronoi region of $\mathbf{x}_{i}$; if the set $W_{i}$ is empty, do nothing; otherwise, compute the average $\mathbf{u}_{i}$ of the set $W_{i}$ and set

$$
\mathbf{x}_{i} \leftarrow \frac{\left(\alpha_{1} j_{i}+\beta_{1}\right) \mathbf{x}_{i}+\left(\alpha_{2} j_{i}+\beta_{2}\right) \mathbf{u}_{i}}{j_{i}+1} \quad \text { and } \quad j_{i} \leftarrow j_{i}+1 ;
$$

the new set of $\left\{\mathbf{x}_{i}\right\}$, along with the unchanged $\left\{\mathbf{x}_{j}\right\}$ corresponding to empty $W_{j}$, form the new set of points $\left\{\mathbf{x}_{i}\right\}_{i=1}^{n}$; if $\Omega$ is a hypersurface, then $\mathbf{x}_{i}$ must be projected onto $\Omega$.

4. If the new points meet some convergence criterion, terminate; otherwise, return to Step 2.

In Steps 1 and 2 of Algorithm 2 as well as in Step 1 of Algorithm 1, points need to be sampled according to a given density function $\rho$. Such sampling steps may be accomplished by a rejection method (Du et al, 2003a; Ju et al, 2002a; Ross, 1998) which we now describe. Given a general domain $\Omega$ in the plane or on the sphere, determine an enclosing rectangle $D$ whose sides are parallel to the coordinate axes or, on the sphere, are latitude and longitude lines, and which contains all points in $\bar{\Omega}$. Set $\rho_{\max }=\max _{\mathbf{x} \in \Omega} \rho(\mathbf{x})$. Then, there are two rejection tests applied. First,

\footnotetext{
12 This algorithm can also be viewed as a generalization of MacQueen's method (MacQueen, 1967); see (Ju et al, 2002a). In fact, if in (5), $q=1, \alpha_{2}=\beta_{1}=0$, and $\alpha_{1}=\beta_{2}=1$, Algorithm 2 reduces to MacQueen's method.
} 
a point $\mathbf{y}$ in $D$ is sampled according to a uniform distribution; ${ }^{13}$ this is done by uniformly sampling each coordinate; all computer systems have a built-in uniform random sampling method. If the sampled point $\mathbf{y}$ is not in $\bar{\Omega}$, it is rejected and one samples again. If the sampled point is in $\bar{\Omega}$, a scalar $\phi$ is sampled uniformly in the interval $[0,1]$. If $\phi<\rho(\mathbf{y}) / \rho_{\max }$, then the sample point is accepted; otherwise, it is rejected. ${ }^{14}$

In Step 3 of Algorithm 2, the average $\mathbf{u}_{i}$ of the sampled points in $W_{i}$ is given by

$$
\mathbf{u}_{i}=\frac{\sum_{\mathbf{y} \in W_{i}} \mathbf{y}}{\# W_{i}},
$$

where $\# W_{i}$ denotes the number of elements in $W_{i}$. Since the points in $W_{i}$ are randomly selected points in the Voronoi region corresponding to $\mathbf{x}_{i}$, one may view $\mathbf{u}_{i}$ as a probabilistic approximation to the centroid (or constrained centroid) of $V_{i}$; the larger is $q$, the better the centroid approximations. ${ }^{15}$ Note that $j_{i}$ keeps track of the number of times that $\mathbf{x}_{i}$ has been previously updated. Some over-relaxation updating methods can be defined by appropriately choosing $\left\{\alpha_{1}, \alpha_{2}, \beta_{1}, \beta_{2}\right\}$; see (Ju et al, 2002a).

Algorithm 2 is much easier to implement and code than Algorithm 1. For Algorithm 1, one has to explicitly construct Voronoi tessellations and determine centers of mass of Voronoi regions. These steps are doable in two-dimensional settings such as planar regions and regions on the sphere and in three-dimensional volumes, but involve considerable coding. On general surfaces in three-dimensions, algorithms for Voronoi tessellations are not generally available and in regions in four and higher dimensions, the calculation of centers of mass become impractical. On the other hand, to find the generators of a CVT, Algorithm 2 does not require the construction of Voronoi tessellations or of centers of mass; both are approximated via sampling. Thus Algorithm 2 can be applied to regions and hypersurfaces in arbitrary dimensions.

The accuracy of Algorithm 1 is limited only by machine precision, although, in practice, one would not want to iterate to that level of accuracy. On the other hand, for Algorithm 2, accuracy is limited by the sampling errors made in Step 2. The $q$ sampled points are divided among the generators so that, say, in a uniform density setting, each generator would only be assigned roughly $q / n$ points, where $n$ denotes the number of generators. Thus, if, say, Monte Carlo sampling is used, the errors in the probabilistic approximations of the centroids of the Voronoi regions would be proportional to $\sqrt{n / q}$ so that this is the best accuracy one can expect from Algorithm 2. Note that, for fixed $q$, the accuracy degrades as we increase the number

\footnotetext{
13 Instead of random, i.e., Monte Carlo, sampling, one can, in conjunction with the rejection steps, use quasi-Monte Carlo, Latin hypercube, etc. sampling methods (McKay and Beckman, 1979; Niederreiter, 1992; Saltelli et al, 2004) appropriate for hypercubes.

${ }^{14}$ Note that both rejection tests can be incorporated into a single test because an alternate means for rejecting points that are outside of $\bar{\Omega}$ is to simply set $\rho(\mathbf{x})=0$ outside of $\bar{\Omega}$.

${ }^{15}$ If $\alpha_{1}=\beta_{1}=0$, and $\alpha_{2}=\beta_{2}=1$, we have in (5) that $\mathbf{x}_{i} \leftarrow \mathbf{u}_{i}$, i.e., the new generators are probabilistic approximations of the centroid of the Voronoi regions; this justifies saying that Algorithm 2 is a probabilistic generalized Lloyd's method.
} 
of generators $n$ and that, for fixed $n$, greater accuracy can be achieved by increasing the number of sample points $q$. Also, note that it is useless to set a tolerance in whatever stopping criterion is used in Step 4 of Algorithm 2 to be smaller than $O(\sqrt{n / q})$.

Because accuracy control is better served by Algorithm 1, it is usually the algorithm of choice for regions in the plane and on the sphere and for three-dimensional regions. For other cases, e.g., higher-dimensional regions and general surfaces in three dimensions, Algorithm 2 becomes more practical.

We close this section on algorithms for CVT construction by noting that several other schemes for computing CVTs such as Newton-type algorithms and multi-level methods are studied in (Du and Emelianenko, 2006, 2008; Liu et al, 2009).

\subsection{The relation between the density function and the local mesh size}

An interesting problem about the asymptotic behavior CVTs is the distribution of the energy $\mathscr{K}$ defined in (3). It was shown in (Du et al, 1999), that in the onedimensional case, for the CVT of $n$ generators $\left\{\left(\mathbf{x}_{i}, V_{i}\right)\right\}_{i=1}^{n}$ with a smooth density function $\rho$, we have

$$
\mathscr{K}_{i} \approx \frac{1}{12} \rho\left(\mathbf{x}_{i}\right) h_{i}^{3} \approx \frac{\mathscr{K}}{n}, \quad \forall 1 \leq i \leq n,
$$

where $h_{i}$ denotes the diameter of $V_{i}, \mathscr{K}_{i}=\int_{V_{i}} \rho(\mathbf{x})\left\|\mathbf{x}-\mathbf{x}_{i}\right\|^{2} \mathrm{~d} \mathbf{x}$, and $\mathscr{K}=\sum_{i=1}^{n} \mathscr{K}_{i}$, i.e., under some assumptions on the density function, asymptotically speaking, the energy is equally distributed in the Voronoi intervals and the diameter of Voronoi intervals are inversely proportional to the one-third power of the underlying density. Based on (6) and the fact $\sum_{i=1}^{n} h_{i}=$ length of $\Omega$, we then obtain an approximation of total clustering energy of the CVT in one dimension given by, for $n$ large,

$$
\mathscr{K} \approx \frac{1}{12} \frac{\left(\int_{\Omega} \rho^{1 / 3} \mathrm{~d} \mathbf{x}\right)^{3}}{n^{2}} .
$$

Let $d$ denote the space dimension and set $d^{\prime}=d-1$ if $\Omega$ is a hypersurface and $d^{\prime}=d$ otherwise. For higher dimensions, a similar conjecture about CVTs or CCVTs can be stated as follows:

$$
\begin{aligned}
& \mathscr{K}_{i} \approx c_{1} \rho\left(\mathbf{x}_{i}\right) h_{i}^{d^{\prime}+2} \approx \frac{\mathscr{K}}{n}, \quad \forall 1 \leq i \leq n, \\
& \mathscr{K} \approx \frac{c_{2}}{n^{2 / d^{\prime}}}\left(\int_{\Omega} \rho^{d^{\prime} /\left(d^{\prime}+2\right)} \mathrm{d} \mathbf{x}\right)^{\left(d^{\prime}+2\right) / d^{\prime}},
\end{aligned}
$$


where $c_{1}, c_{2}$ are constants depending only on $d^{\prime}$. This conjecture still remains open for $d \geq 2$ although its validity has been supported through many numerical studies and widely used for applications in vector quantizations (Gersho and Gray, 1992) and image processing.

A direct consequence of (7) is

$$
\frac{h_{i}}{h_{j}} \approx\left(\frac{\rho\left(\mathbf{x}_{j}\right)}{\rho\left(\mathbf{x}_{i}\right)}\right)^{1 /\left(d^{\prime}+2\right)} .
$$

The relation (9) between the density function and the local mesh sizes is also very useful in CVT-based adaptive mesh generation and optimization (Ju, 2007; Ju et al, 2002b).

\section{Application to climate and global modeling}

\subsection{Global SCVT meshes}

We define quantitative measures of grid quality that we can use to assess the quality of meshing schemes on the sphere. let

Given a Voronoi mesh $\left\{\left(\mathbf{x}_{i}, V_{i}\right)\right\}_{i=1}^{n}$, set $Q=\left\{(i, j) \mid \mathbf{x}_{i}\right.$ and $\mathbf{x}_{j}$ are neighbors $\}$ and

$$
h_{\min }=\min _{(i, j) \in Q}\left\|\mathbf{x}_{i}-\mathbf{x}_{j}\right\| \quad \text { and } \quad h_{\max }=\max _{(i, j) \in Q}\left\|\mathbf{x}_{i}-\mathbf{x}_{j}\right\| .
$$

Clearly, the ratio (Du et al, 2003b)

$$
\mu=\frac{h_{\max }}{h_{\min }}
$$

is a natural measurement of the global uniformity of the Voronoi mesh $\left\{\mathbf{x}_{i}, V_{i}\right\}_{i=1}^{n}$. It is clear that $\mu \geq 1$ and the smaller is $\mu$, the more globally uniform is the Voronoi mesh.

Letting $\chi_{i}$ denote the set of neighbor generators of $\mathbf{x}_{i}$, a measure of the local quality or local uniformity of the Voronoi mesh at $\mathbf{x}_{i}$ is given by

$$
\sigma_{i}=\frac{\min _{j \in \chi_{i}}\left\|\mathbf{x}_{i}-\mathbf{x}_{j}\right\|}{\max _{j \in \chi_{i}}\left\|\mathbf{x}_{i}-\mathbf{x}_{j}\right\|} .
$$

Clearly $0<\sigma_{i} \leq 1$ and the larger is $\sigma$, the better the local uniformity.

We apply the commonly used $q$-measure (Field, 2000) to evaluate the quality of dual Delaunay triangular meshes, where, for any triangle $T_{i}, q_{i}$ is defined to be twice the ratio of the radius $R_{T_{i}}$ of the largest inscribed circle and the radius $r_{T_{i}}$ of the smallest circumscribed circle, i.e., 


$$
q_{i}=2 \frac{R_{T_{i}}}{r_{T_{i}}}=\frac{(b+c-a)(c+a-b)(a+b-c)}{a b c},
$$

where $a, b$, and $c$ denote the side lengths of $T_{i}$. Clearly, $0<q_{i} \leq 1$ and $q_{i}=1$ corresponds to the equilateral triangle.

We then define the mesh quality measures

$$
\sigma_{\min }=\min _{i=1, \ldots, m_{D}} \sigma_{i}, \quad \sigma_{\text {avg }}=\frac{1}{n} \sum_{i=1}^{m_{d}} \sigma_{i}, \quad q_{\min }=\min _{i=1, \ldots, m_{D}} q_{i}, \quad q_{\text {avg }}=\frac{1}{m_{D}} \sum_{i=1}^{m_{d}} q_{i},
$$

where $m_{D}$ denotes the number of dual Delaunay triangles. The closer these measures are to unity, the better the mesh.

\subsubsection{Uniform SCVT meshes vs. icosahedral-bisection meshes}

Icosahedral-bisection meshes on the sphere have been widely used in the climate and global modeling communities; icosahedral-bisection meshes from a family of hierarchical meshes with $10 \times 4^{\ell-1}+2$ nodes at level $\ell$, in which there are 12 pentagons and all others cells are hexagons. The level $\ell=1$ and $\ell=2$ meshes having 12 and 42 nodes, respectively, are SCVT meshes with respect to the uniform density, but all other members of the family with levels $l>2$ are not SCVTs, although they are quite uniform. We use the centroids of the Voronoi cells of each icosahedralbisection mesh as the initial guess and apply Lloyd's method with a uniform density to generate a sequence of SCVT meshes; see Figure 7. The quality measures of Section 4 for the icosahedral-bisection and uniform SCVT meshes are given in Table 1. The SCVT meshes do better with respect to the local mesh quality measures, i.e., with respect to local mesh uniformity, although they get worse with respect to global mesh uniformity due to the shrinking relative size of the pentagonal cells as the mesh size decreases.

\subsubsection{Locally refined SCVT meshes}

Let a point $\mathbf{x}$ on the sphere be represented by its spherical coordinate $\mathbf{x}=($ lat, lon $)$ with $-\pi / 2 \leq$ lat $\leq \pi / 2$ and $0 \leq$ lon $<2 \pi$. Set $\mathbf{x}_{c}=(\pi / 6,3 \pi / 2)$ and define

$$
d_{S}\left(\mathbf{x}, \mathbf{x}_{c}\right)=\sqrt{(\text { lat }-\pi / 6)^{2}+(\text { lon }-3 \pi / 2)^{2}} .
$$

Define the subregion of the sphere

$$
S_{m t}=\left\{\mathbf{x}=(\text { lat }, \text { lon }) \mid d_{s}\left(\mathbf{x}, \mathbf{x}_{c}\right) \leq \pi / 6\right\} .
$$

In the subregion, we want a high-quality mesh having a local mesh size that is $\gamma_{s}$ times smaller than that outside the subregion. We also want a smooth transition between the coarse and fine grid regions. 

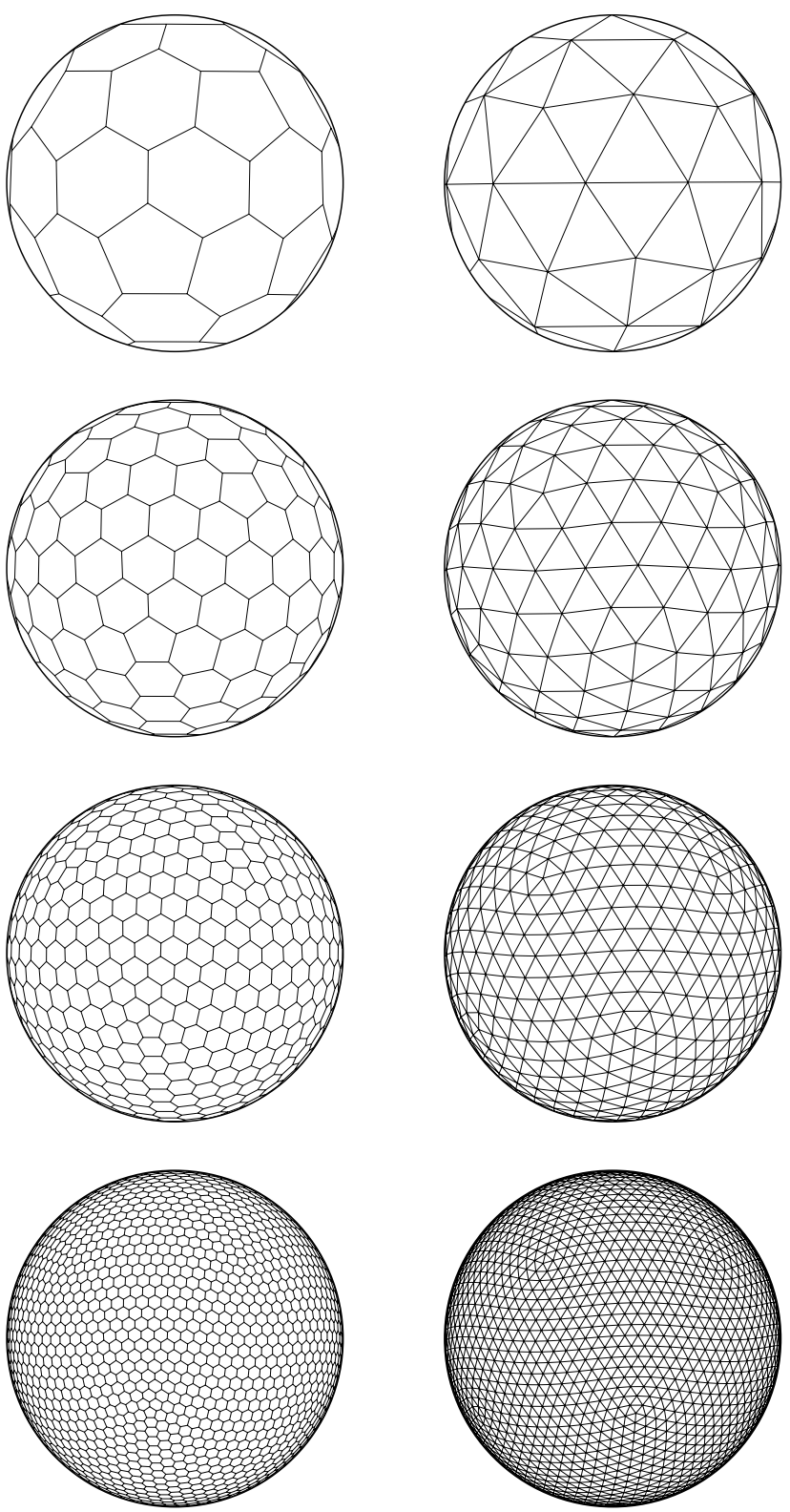

Fig. 7 From top to bottom: spherical centroidal Voronoi tessellations (left column) with 42, 162, 642, 2562 generators for a uniform density and the corresponding spherical Delaunay triangulations (right column). 


\begin{tabular}{|c|c|c||c|c|c|c|c|}
\hline Level $\ell$ & \# of generators & Mesh types & $\mu$ & $\sigma_{\text {avg }}$ & $\sigma_{\min }$ & $q_{\text {avg }}$ & $q_{\min }$ \\
\hline \hline 2 & \multirow{2}{*}{42} & I-B & 1.1308 & 0.9174 & 0.8843 & 0.9872 & 0.9829 \\
\cline { 3 - 7 } & & SCVT & 1.1308 & 0.9174 & 0.8843 & 0.9872 & 0.9829 \\
\hline 3 & \multirow{2}{*}{162} & I-B & 1.1777 & 0.9111 & 0.8586 & 0.9904 & 0.9729 \\
\cline { 3 - 7 } & & SCVT & 1.1647 & 0.9174 & 0.8843 & 0.9872 & 0.9829 \\
\hline 4 & \multirow{2}{*}{642} & I-B & 1.1907 & 0.8737 & 0.8482 & 0.9865 & 0.9701 \\
\cline { 3 - 7 } & & SCVT & 1.1592 & 0.9121 & 0.8525 & 0.9923 & 0.9701 \\
\hline 5 & \multirow{2}{*}{2562} & I-B & 1.1940 & 0.8803 & 0.8405 & 0.9866 & 0.9694 \\
\cline { 3 - 7 } & & SCVT & 1.2335 & 0.9141 & 0.8511 & 0.9931 & 0.9694 \\
\hline 6 & \multirow{2}{*}{10242} & I-B & 1.1948 & 0.8879 & 0.8386 & 0.9866 & 0.9692 \\
\cline { 3 - 7 } & & SCVT & 1.2710 & 0.9157 & 0.8507 & 0.9934 & 0.9692 \\
\hline 7 & \multirow{2}{*}{40962} & I-B & 1.1951 & 0.8932 & 0.8380 & 0.9866 & 0.9692 \\
\cline { 3 - 7 } & & SCVT & 1.3107 & 0.9168 & 0.8504 & 0.9935 & 0.9692 \\
\hline 8 & \multirow{2}{*}{163842} & I-B & 1.1951 & 0.8966 & 0.8379 & 0.9870 & 0.9692 \\
\cline { 3 - 7 } & & SCVT & 1.3526 & 0.9173 & 0.8494 & 0.9952 & 0.9687 \\
\hline 9 & \multirow{2}{*}{655362} & I-B & 1.1952 & 0.8970 & 0.8378 & 0.9952 & 0.9691 \\
\cline { 3 - 7 } & & SCVT & 1.4080 & 0.9167 & 0.8465 & 0.9987 & 0.9675 \\
\hline
\end{tabular}

Table 1 Comparisons of quality of icosahedral-bisection (I-B) and uniform spherical centroidal Voronoi tessellation (SCVT) meshes.

Using the density-mesh size relation (9), the density function is set to

$$
\rho(\mathbf{x})= \begin{cases}\gamma_{s}^{4} & \text { if } d_{s}\left(\mathbf{x}, \mathbf{x}_{c}\right) \leq \pi / 6 \\ \left(\left(1-s_{\mathbf{x}}\right) \gamma_{s}+s_{\mathbf{x}}\right)^{4} & \text { if } \pi / 6<d_{s}\left(\mathbf{x}, \mathbf{x}_{c}\right) \leq \pi / 6+\varepsilon_{s} \\ 1 & \text { otherwise, }\end{cases}
$$

where $\varepsilon_{s}$ denotes the width of the transition layer and $s_{\mathbf{X}}=\frac{d_{s}\left(\mathbf{x}, \mathbf{x}_{c}\right)-\pi / 6}{\varepsilon_{s}}$; we set $\gamma_{s}=3$ and $\varepsilon_{s}=\pi / 12$ here. The resulting SCVT with 2562 generators produced by Lloyd's method and the corresponding dual Delaunay triangulation are presented in Figure 8 (top row). Variations in the Voronoi cell sizes and areas are plotted in Figure 8 (bottom row). The histogram of the size distribution clearly indicates that there are two dominant mesh sizes; cells 1 through 1250 have one size, cells 1500 through 2500 have another size, and these two cells sizes differ by a factor of three as predicted by (9). For this example, we have $\mu=5.4018, \sigma_{\text {avg }}=0.8712, \sigma_{\min }=0.4533$, $q_{\text {avg }}=0.9854$, and $q_{\min }=0.6886$.

Figure 8 as well as Figure 9 below illustrate an important feature of nonuniform CVT and SCVT grids, namely smooth transitions from coarse to fine grids. This can always be effected within the CVT/SCVT framework through the use of smooth density functions so that, if a given density function is not smooth, it is often beneficial to smooth it before using it to generate CVT/SCVT grids; see Section 4.2.

\subsubsection{Nested SCVT meshes}

For this example, the region of interest covers most of North America, i.e., 

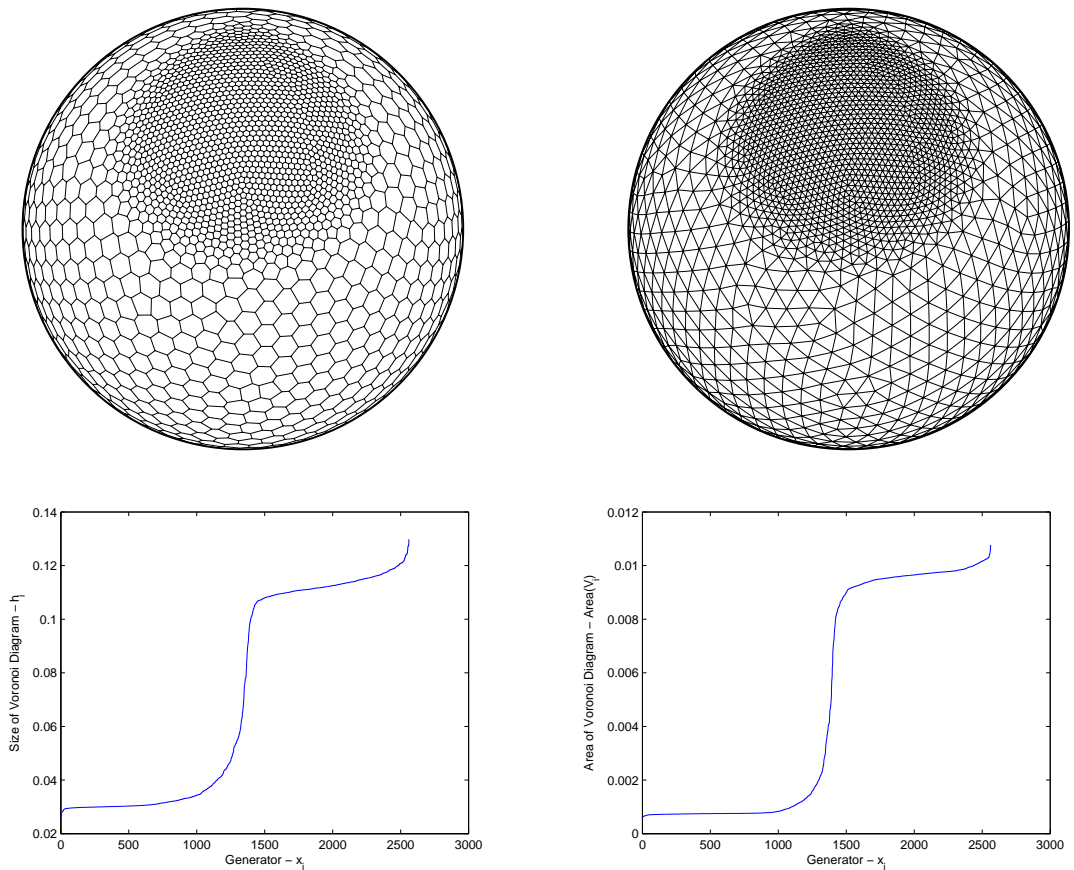

Fig. 8 Top row: a spherical centroidal Voronoi tessellation (left) and its dual spherical Delaunay triangulation (right) with 2562 generators and the density (12); bottom row: plot of Voronoi cell sizes (left) and areas (right).

$$
S_{n \ell}=\left\{\mathbf{x}=(\text { lat }, \text { lon }) \mid-5^{\circ} \leq \text { lat } \leq 60^{\circ}, \quad 225^{\circ} \leq \text { lat } \leq 310^{\circ}\right\} .
$$

Again, we want a high-quality mesh with local mesh size in $S_{n \ell}$ being approximately $\gamma_{s}=3$ times smaller that that in outside that region. This time we use a different means to generate a locally refined SCVT mesh because we wish to make use of global uniform SCVT meshes.

We begin with the global uniform SCVT with 2562 nodes shown in Section 4.1.1. The submesh falling inside $S_{n \ell}$ has about 355 nodes. We refine this submesh to get a new mesh of $S_{n \ell}$ with 3574 nodes (about 10 times more nodes). We then merge the refined submesh with the remaining generators of the original unform SCVT outside of $S_{n \ell}$ and produce a new global nonuniform Voronoi mesh with 5781 generators; the result is clearly not a SCVT but we use it as an initial guess for Lloyd's method. We choose a Similar to (12), we choose the density function

$$
\rho(\mathbf{x})= \begin{cases}\gamma_{s}^{4} & \text { if } \mathbf{x} \in S_{n \ell} \\ \left(\left(1-s_{\mathbf{x}}\right) \gamma_{s}+s_{\mathbf{x}}\right)^{4} & \text { if } 0<d\left(\mathbf{x}, S_{n \ell}\right) \leq \varepsilon_{s} \\ 1 & \text { otherwise, }\end{cases}
$$


where $s_{\mathbf{X}}=\frac{d\left(\mathbf{x}, S_{n \ell}\right)}{\varepsilon_{\varepsilon}}$ and the width of the transition layer $\varepsilon_{s}=0.24$. Then, we apply Lloyd's method with this density, adding one more restriction: all generators $\mathbf{x}_{i}$ are fixed during the iterations if $d\left(\mathbf{x}_{i}, S_{n \ell}\right)>\varepsilon_{s}$.

The resulting SCVT with 5781 generators and its dual Delaunay triangulation are presented in Figure 9 (top row). Variations of Voronoi cell sizes and areas are plotted in the bottom row. For this example, we have $\mu=5.7079, \sigma_{\text {avg }}=0.9006$, $\sigma_{\text {min }}=0.4012, q_{\text {avg }}=0.9904$ and $q_{\min }=0.7114$.
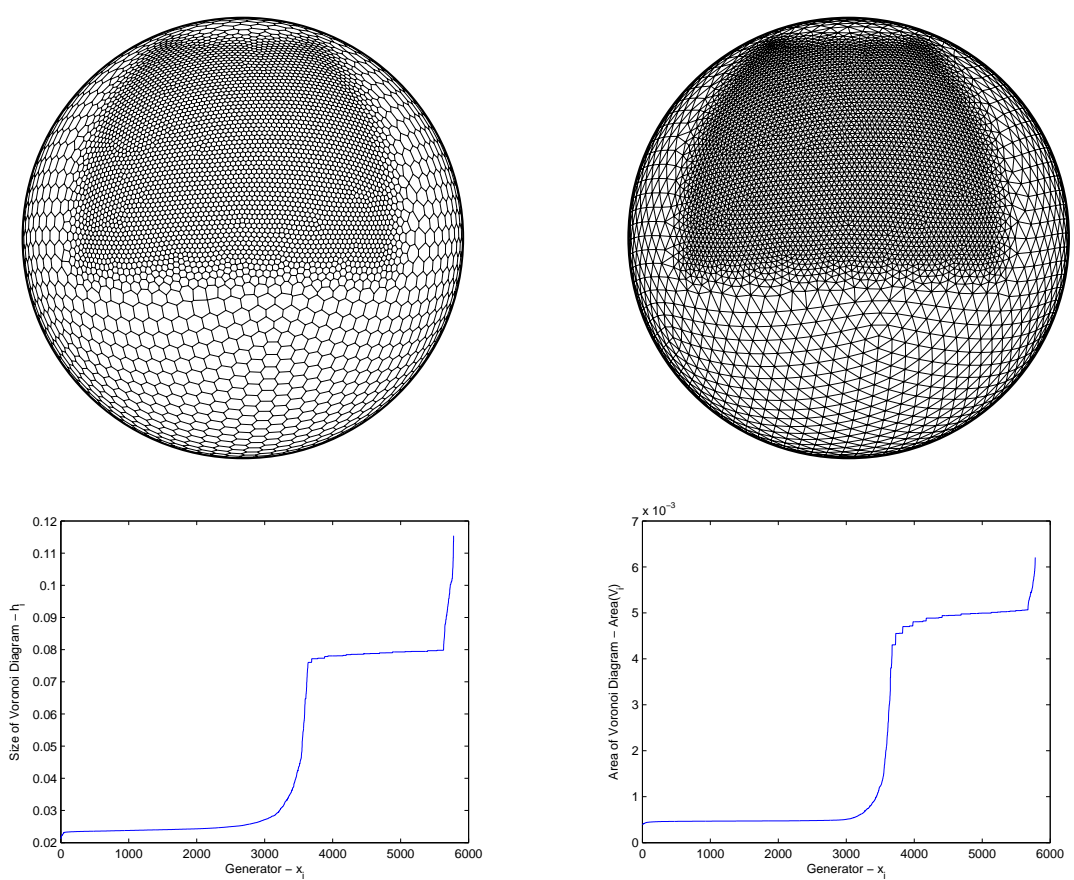

Fig. 9 Top row: a spherical centroidal Voronoi tessellation (left) with 5781 generators and its dual spherical Delaunay triangulation (right) produced by the nested method; bottom row: plot of Voronoi cell sizes (left) and areas (right).

\subsection{CVT-based regional meshes of the North Atlantic Ocean}

Figure 10 (top left) shows the time-mean kinetic energy from a global 0.1 degree simulation of the North Atlantic Ocean (Smith et al, 2000). We use this data set to determine both the boundary of the North Atlantic ocean and an appropriate density 
function, and then construct the CVT mesh based on this information; see (Ringler et al, 2008) for details. ${ }^{16}$

Based on the kinetic energy, $K E$, we defined the density function

$$
\rho=\max \left[0.1, \frac{K E}{K E_{\max }}\right]^{4},
$$

where $K E_{\max }$ is the maximum kinetic energy in the domain. The lower bound 0.1 insures that the grid in quiescent regions is not overly coarse. We also raise the value of the density function as we approach the boundary of the ocean so that the boundary is resolved to a desired resolution; this is accomplished by making the density in regions near a land boundary also depend in an inverse manner on the distance to the boundary. The resulting mesh has a grid spacing that varies by a factor of 10 .

In order to allow for a smooth transition between regions of high and low resolution, we apply a substantial amount, e.g., approximately 20 passes, of Laplacian smoothing ${ }^{17}$ to our density function. Figure 10 shows some of the resulting CVT meshes. Whereas the two examples given above produce a mesh with two dominant resolutions, in this example a wide spectrum of resolutions are present. Note that this type of mesh will lead to additional complications related to parameter settings of sub-grid closures but that is also offers the opportunity to adaptively select multiple closure models whose efficacy depends on the local grid size. All in all, variational resolution meshes such as the one illustrated in Figure 10 are significantly more ambitious than those considered in Sections 4.1.2 and 4.1.3.

\subsection{Numerical simulations with SCVT meshes}

\subsubsection{Mesh decomposition for parallel computing}

We take a global SCVT mesh with 40962 generators (about $120 \mathrm{~km}$ resolution) and separate it into 642 blocks; see Figure 11. These blocks are created so as to balance the work-per-block and to minimize the amount of information that must be communicated between blocks; the software package "METIS" (Karypis and Kumar, 1998) in which a family of multilevel partitioning algorithms is implemented is used for this purpose. We can assign an arbitrary number of blocks per processor so that two types of parallelism within are supported within this framework, i.e., distributed memory across nodes and shared memory within a node.

\footnotetext{
${ }^{16}$ In practice, we would not use such a proxy to determine a variable resolution CVT grid, but instead would adaptively determine the grid from the simulation model output.

${ }^{17}$ In the current context, Laplacian smoothing is a process of smoothing the a function defined on a grid. One replaces the value of a function at a point by first averaging its value at neighboring points and then averaging that result with its own value at the point.
} 

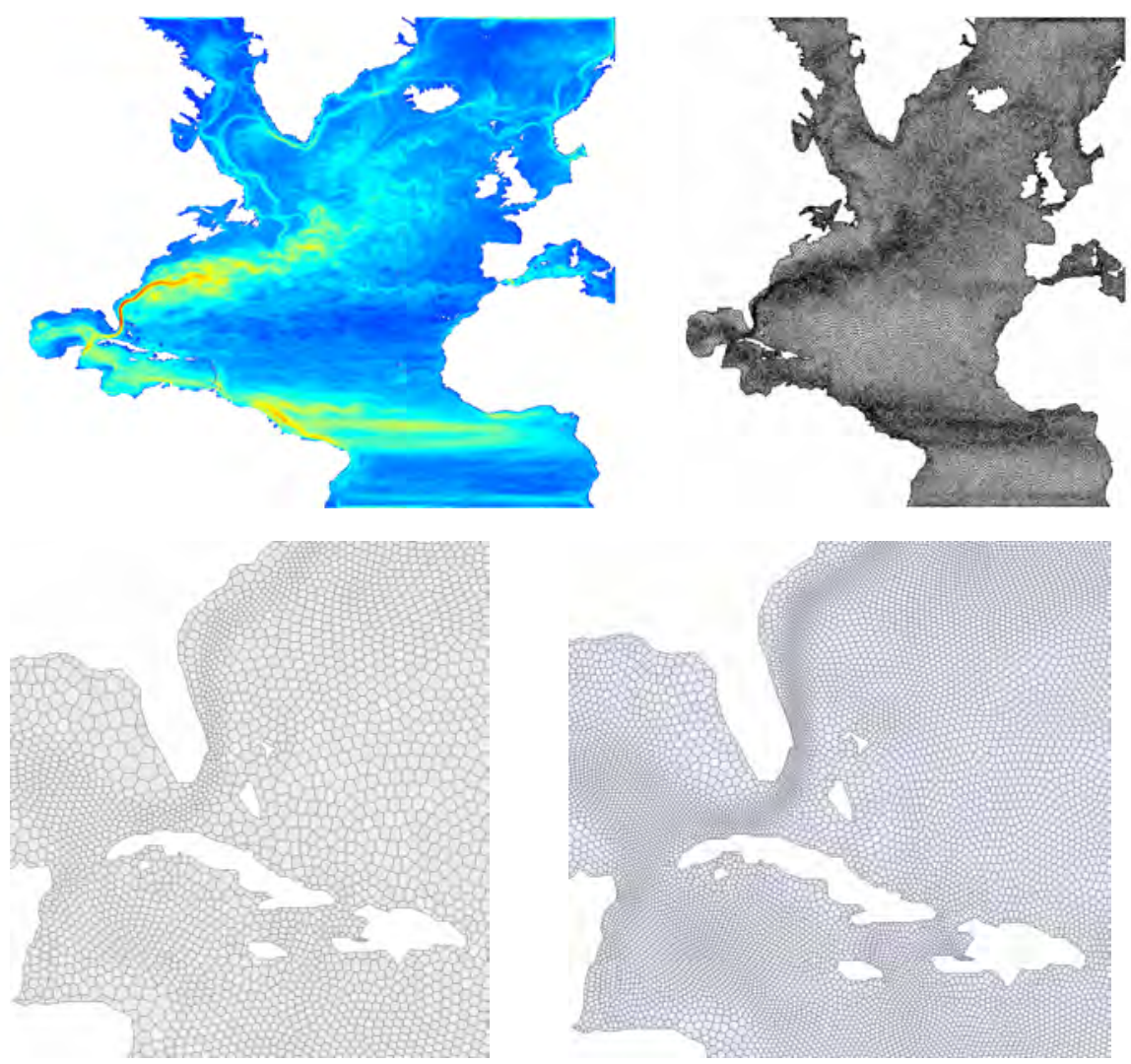

Fig. 10 Top-left: time-mean kinetic energy of the North Atlantic Ocean; top-right: a CVT mesh with 47305 generators of the North Atlantic; bottom-left: a zoom-in of the CVT mesh; bottomright: a zoom-in of the same region of a CVT mesh with 183907 generators.

\subsubsection{Example numerical methods}

As discussed in Section 1, all typical finite-volume grid staggerings used for quadrilateral meshes, i.e., A-, B-, C- and Z-grid staggerings, have been successfully applied to Voronoi tessellations. C-grid staggering has shown promising results, particularly when applied to variable resolution meshes. See Check: Ringler Dyncore Chapter for a broad discussion of C-grid staggerings and see (Thuburn et al, 2009; Ringler et al, 2010) for an in-depth discussion of C-grid staggering applied to the nonlinear shallow-water equations.

We apply the methods developed in (Thuburn et al, 2009; Ringler et al, 2010) to test case 5 of the standard shallow-water test cases developed in (Williamson et al, 2001). A flow in geostrophic balance is confronted with a large-scale orographic feature at the start of the simulation, $t=0$. The transient forcing at $t=0$ leads to the generation of large-amplitude gravity waves and Rossby waves. The sole forcing 


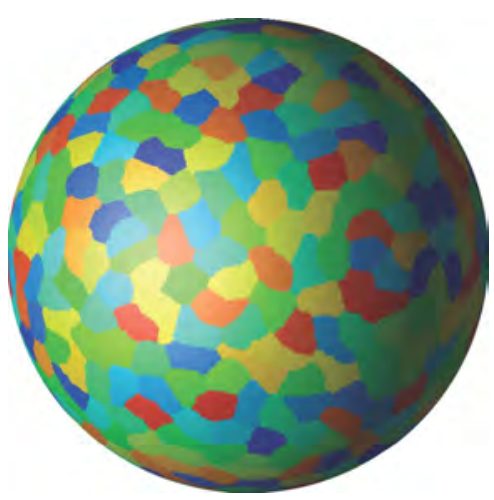

Fig. 11 Decomposition of a global SCVT mesh of 40962 generators into 642 blocks. The blocks can be distributed across computational nodes for implementation on high-performance architectures.

mechanism is the presence of the orographic forcing. While no analytical solution is known, results from high-resolution global spectral models (Lipscomb and Ringler, 2005) are adequate reference solutions for the simulations conducted here.

Figure 12 shows the potential vorticity and kinetic energy at day 50 when using a SCVT with 40962 cells based on a uniform density function. Shallow-water test case 5 is shown to breakdown into 2D turbulence after day 25, so Figure 12 shows a snapshot of this turbulent behavior. Even in the presence of fully-developed 2D turbulence, the simulation is stable and robust while conserving total energy to within time truncation error. Simulations of this same test case, but using the variable resolution meshes shown in Figures 8 and 9, produce equally robust results.
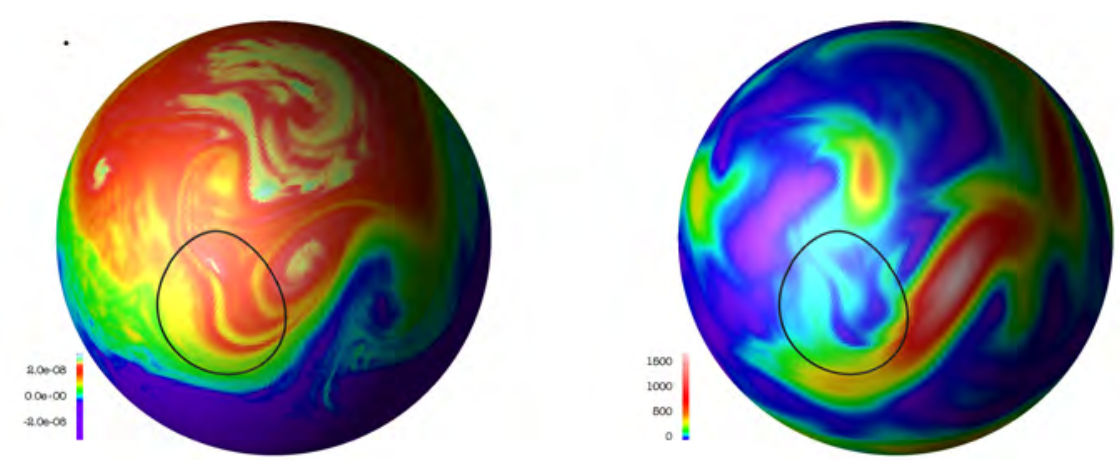

Fig. 12 Simulation results at day 50 using a uniform SCVT mesh with the method outlined in (Ringler et al, 2010). The figure depicts the potential vorticity field (left) and the kinetic energy field (right). The simulation conserves potential vorticity to machine precision and total energy to within time-truncation error 


\section{Summary}

Voronoi tessellations and, in particular, centroidal Voronoi tessellations, offer a robust approach to tiling the surface of the sphere. The Delaunay triangulation is the dual of the Voronoi tessellations, so whether hexagons or triangles are of interest, this approach will result in high-quality uniform and nonuniform meshes. Centroidal Voronoi tessellations are particularly well-suited for the generation of smoothly varying meshes, thus providing a possible alternative to traditional nesting approaches. With the recent discovery of a class of finite-volume methods that are directly applicable to variable resolution meshes ((Thuburn et al, 2009; Ringler et al, 2010)), it appears that the creation of variable resolution, global climate system models is now possible.

Acknowledgements This work was supported by the US Department of Energy Office of Science Climate Change Prediction Program through grant numbers DE-FG02-07ER64431 and DE-FG0207ER64432, and the US National Science Foundation under grant numbers DMS-0609575 and DMS-0913491.

The authors thank the reviewers and editors for the many helpful comments that resulted in substantial improvements to this chapter.

\section{References}

Alliez P, Cohen-Steiner D, Yvinec M, Desbrun M (2005) Variational tetrahedral meshing. In: Proceedings of SIGGRAPH, pp 617-625

Arakawa A (1966) Computational design for long-term numerical integration of the equations of fluid motion: Two-dimensional incompressible flow. J Comput Phys 1:119-143

Arakawa A, Lamb V (1977) Compuational design of the basic dynamical processes in the UCLA general circulation model. J Comput Phys 17:173-265

Arakawa A, Lamb V (1981) A potential enstrophy and energy conserving scheme for the shallow water equations. Mon Wea Rev 109:18-36

Augenbaum J (1984) A lagrangian method for the shallow water equations based on a voronoi mesh - one dimensional results. J Comput Phys 53:240-265

Augenbaum J, , Peskin C (1985) On the construction of the Voronoi mesh on the sphere. J Comput Phys 59:177-192

Barnes E, Sloane N (1983) The optimal lattice quantizer in three dimensions. SIAM J Algeb Disc Meth 4:31-40

Bentley J, Weide B, Yao A (1980) Optimal expected-time algorithms for cloest point problems. ACM Trans Math Soft 6:563-580

Boissonnat J, Oudot S (2005) Provably good sampling and meshing of surfaces. Graph Models 67:405-451

Chynoweth S, Sewell M (1990) Mesh duality and Legendre duality. Proc R Soc London A 428:351-377 
Cignoni P, Montani C, Scopigno R (1998) Dewall: A fast divide and conquer delaunay triangulation algorithm in $e^{d}$. Computer-Aided Design 30:333-341

Cooley J, Tukey J (1965) An algorithm for the machine calculation of complex Fourier series. Math Comp 19:197-301

Delaunay B (1928) Sur la sphère vide. In: Proceedings of the International Mathematical Congress, pp 695-700

Delaunay B (1934) Sur la sphère vide. Izvestia Akademii Nauk SSSR, Otdelenie Matematicheskikh i Estestvennykh Nauk 7:793-800

Dirichlet G (1850) Uber die Reduktion der positiven quadratischen Formen mit drei unbestimmten ganzen Zahlen. J Reine Angew Math 40:209-227

Du Q, Emelianenko M (2006) Acceleration schemes for computing the centroidal Voronoi tessellations. Numer Linear Alg Appl 13:173-192

Du Q, Emelianenko M (2008) Uniform convergence of a nonlinear energy-based multilevel quantization scheme via centroidal Voronoi tessellations. SIAM J Numer Anal 46:1483-1502

Du Q, Ju L (2005) Finite volume methods on spheres and spherical centroidal Voronoi meshes. SIAM J Numer Anal 43:1673-1692

Du Q, Wang D (2003) Tetrahedral mesh generation and optimization based on centroidal Voronoi tessellations. Int J Numer Methods Engrg 56:1355-1373

Du Q, Wang D (2005) On the optimal centroidal Voronoi tessellations and Gersho's conjecture in the three dimensional space. Comput Math Appl 49:1355-1373

Du Q, Faber V, Gunzburger M (1999) Centroidal Voronoi tessellations: Applications and algorithms. SIAM Review 41:637-676

Du Q, Gunzburger M, Ju L (2003a) Constrained centroidal Voronoi tessellations for surfaces. SIAM J Sci Comput 24:1488-1506

Du Q, Gunzburger M, Ju L (2003b) Voronoi-based finite volume methods, optimal Voronoi meshes and PDEs on the sphere. Comput Meth Appl Mech Engrg 192:3933-3957

Du Q, Emelianenko M, Ju L (2006) Convergence of the lloyd algorithm for computing centroidal Voronoi tessellations. SIAM J Numer Anal 44:102-119

Edelsbrunner H, Shah N (1996) Incremental topological flipping works for regular triangulations. Algorithmica 15:223-241

Emelianenko M, Ju L, Rand A (2008) Nondegeneracy and weak global convergence of the Lloyd algorithm in $\mathbb{R}^{d}$. SIAM J Numer Anal 46:1423-1441

Field D (2000) Quantitative measures for initial meshes. Int J Numer Meth Engrg 47:887-906

Fortune S (1986) A sweepline algorithm for Voronoi diagrams. In: Proceedings of the Second Ann. Symp. Comput. Geom., pp 313-322

Freitag L, Ollivier-Gooch C (1997) Tetrahedral mesh improvement using swapping and smoothing. Int J Numer Methods Engrg 40:3979-4002

Gersho A (1979) Asymptotically optimal block quantization. IEEE Trans Inform Theory 25:373-380

Gersho A, Gray R (1992) Vector Quantization and Signal Compression. Kluwer, Boston 
Guiba L, Knuth D, Sharir M (1992) Randomized incremental construction of Delaunay and Voronoi diagrams. Algorithmica 7:381-413

Guibas L, Stolfi J (1985) Primitives for the manipulation of general subdivisions and the computation of Voronoi diagrams. ACM Trans Graphics 4:74-123

Hartigan J (1975) Clustering Algorithms. Wiley, New York

Heikes R, Randall D (1995a) Numerical integration of the shallow-water equations on a twisted icosahedral grid. Part i: basic design and results of tests. Mon Wea Rev 123:1862-1880

Heikes R, Randall D (1995b) Numerical integration of the shallow-water equations on a twisted icosahedral grid. Part ii. a detailed description of the grid and an analysis of numerical accuracy. Mon Wea Rev 123:1881-1887

Ju L (2007) Conforming centroidal Voronoi Delaunay triangulation for quality mesh generation. Inter J Numer Anal Model 4:531-547

Ju L, Du Q, Gunzburger M (2002a) Probabilistic methods for centroidal Voronoi tessellations and their parallel implementations. Para Comput 28:1477-1500

Ju L, Gunzburger M, Zhao W (2002b) Adaptive finite element methods for elliptic PDEs based on conforming centroidal Voronoi-Delaunay triangulations. SIAM J Sci Comput 28:2023-2053

Kanungo T, Mount D, Netanyahu N, Piatko C, Silverman R, Wu A (2002) An efficient k-means clustering algorithm: Analysis and implementation. IEEE Trans Pattern Anal Mach Intel 24:881-892

Karypis G, Kumar V (1998) A fast and high quality multilevel scheme for partitioning irregular graphs. SIAM J Sci Comput 20:359-392

Kasahara A, Washington W (1967) NCAR global general circulation model of the atmosphere. Mon Wea Rev 95:389-402

Kittel C (2004) Introduction to Solid State Pgysics. Wiley, New York

Lee D, Schachter B (1980) Two algorithms for constructing a Delaunay triangulation. Int J Inform Sci 9:219-242

Lipscomb W, Ringler T (2005) An incremental remapping transport scheme on a spherical geodesic grid. Mon Wea Rev 133:2335-2350

Liu Y, Wang W, Levy B, Sung F, Yan DM (2009) On centroidal Voronoi tessellation - Energy smoothness and fast computation. ACM Trans Graphics 28:1-17

Lloyd S (1982) Least squares quantization in PCM. IEEE Trans Inform Theory 28:129-137

MacQueen J (1967) Some methods for classification and analysis of multivariate observations. In: Proceedings of the Fifth Berkeley Symp. Math. Stat. and Prob., Ed. by L. LeCam and J. Neyman, vol I, pp 181-197

Masuda Y, Ohnishi H (1986) An integration scheme of the primitive equations model with an icosahedral-hexagonal grid system and its application to the shallow water equations. In: Short-and Medium-Range Numerical Weather Prediction, Ed. by T. Matsuno, Universal Academy Press, pp 317-326

McKay M, Beckman WCR (1979) A comparison of three methods for selecting values of input variables in the analysis of output from a computer code. Technometrics 21:239-245

Newman D (1982) The hexagon theorem. IEEE Trans Inform Theo 28:137-139 
Nguyen H, Burkardt J, Gunzburger M, Ju L, Saka Y (2009) Constrained CVT meshes and a comparison of triangular mesh generators. Comp Geom: Theory Appl 42:1-19

Niederreiter H (1992) Random Number Generation and Quasi-Monte Carlo Methods. SIAM, Philadelphia

Okabe A, Boots B, Sugihara K, Chiu S (2000) Spatial Tessellations: Concepts and Applications of Voronoi Diagrams. Second Edition, Wiley, Chichester

Persson PO, Strang G (2004) A simple mesh generator in Matlab. SIAM Rev 46:329-345

Randall D (1994) Geostrophic adjustment and the finite-difference shallow water equations. Mon Wea Rev 122:1371-1377

Renka R (1997) Algorithm 772. STRIPACK: Delaunay triangulation and Voronoi diagrams on the surface of a sphere. ACM Trans Math Soft 23:416-434

Ringler T, Randall D (2002) A potential enstrophy and energy conserving numerical scheme for solution of the shallow-water equations on a geodesic grid. Mon Wea Rev 130:1397-1410

Ringler T, Heikes R, Randall D (2000) Modeling the atmospheric general circulation using a spherical geodesic grid: A new class of dynamical cores. Mon Wea Rev 128:2471-2490

Ringler T, Ju L, Gunzburger M (2008) A multi-resolution method for climate system modeling. Ocean Dynamics 58:475-498

Ringler T, Thuburn J, Klemp J, Skamarock W (2010) A unified approach to energy conservation and potential vorticity dynamics on arbitrarily structured c-grids. J Comput Phys to appear

Rogers C (1964) Packing and Covering. Cambridge University Press, Cambridge

Ross S (1998) A First Course in Probability. Prentice Hall, Englewood Cliffs

Sadourny R, Arakawa A, Mintz Y (1968) Integration of the nondivergent barotropic vorticity equation with an icosahedral-hexagonal grid for the sphere. Mon Wea Rev 96:351-356

Saltelli A, Chan K, Scott E (2004) Sensitivity Analysis. Wiley, Chichester

Sedgewick R (1983) Algorithms. Addison Wesley

Sewell M (2002) Some applications of transformation theory in mechanics. In: Large-Scale Atmosphere-Ocean Dynamics, Ed. by J. Norbury and I. Roulstone, vol II, Chapter 5

Shamos M, Hoey D (1975) Closest-point problems. In: Proceedings ot the Sixteenth IEEE Annual Symposium on Foundations of Computer Science, pp 151-162

Smith R, Maltrud M, Bryan F, Hecht M (2000) Numerical simulation of the north atlantic ocean at $1 / 10^{\circ}$. J Phys Ocean 20:1532-1561

Snow J (1855) On the Mode of Communication of Cholera. Churchill Livingstone, London

Thuburn J, Ringler T, Klemp J, Skamarock W (2009) Numerical representation of geostrophic modes on arbitrarily structured c-grids. J Comput Phys 228:83218335

Tomita H, Tsugawa M, Satoh M, Goto K (2001) Shallow water model on a modified icosahedral geodesic grid by using spring dynamics. J Comput Phys 174:579-613 
Tomita H, Miura H, Iga S, Nasuno T, Satoh M (2005) A global cloud-resolving simulation: Preliminary results from an aqua planet experiment. Geophys Res Lett 32:L08,805

Voronoi G (1907) Nouvelles applications des paramètres continus à la théorie des formes quadratiques. Primiere Mémoire: Sur quelques prepriétés des formes quadratiques positives parfaites. J Reine Angew Math 133:97-178

Voronoi G (1908) Nouvelles applications des paramètres continus à la théorie des formes quadratiques. Deuxième Mémoire: Recherches usr les parallélloèdres primitifs,. J Reine Angew Math 134:198-287

Weaver W, Shannon C (1963) The Mathematical Theory of Communication. University of Illinois Press, Champaign

Weller H (2009) Predicting mesh density for adaptive modelling of the global atmosphere. Philosophical Transactions of the Royal Society A: Mathematical, Physical and Engineering Sciences 367:4523-4542

Weller H, Weller H (2008) A high-order arbitrarily unstructured finite-volume model of the global atmosphere: Tests solving the shallow-water equations. Inter J Numer Meth Fluids 56:1589-1596

Williamson D (1968) Integration of the barotropic vorticity equation on a spherical geodesic grid. Tellus 20:642-653

Williamson D (1970) Integration of the primitive barotropic model over a spherical geodesic grid. Mon Wea Rev 98:512-520

Williamson D, Drake J, Hack J, Jakob R, Swarztrauber P (2001) A standard test set for numerical approximations to the shallow water equations in spherical geometry. J Comput Phys 102:211-224

Ziman J (1979) Principles of the Theory of Solids. Second Ed., Cambridge University Press, Cambridge 